\title{
Examining the Behavior of a Solid Particle Interacting with Circular Obstacles in an Incompressible Flow
}

\author{
Muhammad Yaqoob $^{1}$, Kainat Komal Kayani ${ }^{1}$, Kamran Usman $^{1 *}$ and Muhammad Shahid ${ }^{1}$ \\ ${ }^{1}$ Department of Mathematics, Air University, Islamabad 44000, Pakistan \\ ${ }^{*}$ Corresponding author
}

\begin{abstract}
We have inspected the effects of solid falling particles passing past circular obstacles in particulate flows and the disturbance created in fluid due to such particle motion. Particle interactions with obstacles present inside the domain, with the outer boundary and with the fluid is investigated. Eulerian technique which uses a fixed computational mesh is adopted through which the freely moving particles pass and interact with the surroundings. The coupled system of solid particle and viscous fluid inside the whole domain is numerically handled using fictitious boundary method (FBM). Lubrication collision models are presented to handle particle-obstacle and particle-wall interactions. FEATFLOW, which is an open source multigrid finite element solver, is used to compute the particulate flow. Numerical experiments are performed taking into account different particle positions and different alignments of circular cylinders (obstacles). Effects of particle motion on the drag forces near the obstacles and on the physical behavior of the fluid-particle-obstacle system due to the particle-wall, particle-obstacle and particle-fluid interactions has been analyzed.
\end{abstract}

Keywords: Direct Numerical Simulation; Fictitious Boundary Method; Finite Element Method; Multigrid; Particulate Flow; Sedimentation. 2010 Mathematics Subject Classification: 76M99; 83A05

\section{Introduction}

A lot of naturally occurring and manufacturing processes are visible while examining the interaction of particles in fluid such as agglomeration, multiphase flows, sedimentation and granular flows. In a situation of large number of particles, collisions of a particle with other particles and their interaction with obstacles inside the domain are involved. A particular example can be observed where tiny clay particles and sediments of sand, present in the fluid, flow and interact with the pillars of a bridge in a river. Other practical examples from engineering point of view can be found in fluid interacting with internal rigid boundaries such as in hanging electrical transmission lines and poles, high rise buildings standing inside the atmospheric air. An underlying knowledge is lacking for the study of such particle interactions with obstacles in a domain which results in different formations of particle trajectories and drag analysis across the obstacles followed by the particle collisions in the modeling of particulate flow [1]. To inspect the solid-liquid flows [2] various numerical approaches have been adopted over the last years. The phenomena of particulate flow is mainly modeled by employing two approaches, either the continuum or the discrete approach. Continuum approach considers the whole mass of the particle as a synthetic continuum which works specifically for flow regimes and is based on the solution of the underlying conservation equations [3]. Fast granular flow and steady granular flow concepts have been presented successfully by Chapman, Ogawa and Rao et al. [3], [4]. Recently a level set methodology has been used by Sokolov and coauthors [5] for solving partial differential equations coupled with manifold/surfaces. The other approach, known as the discrete approach, takes into account the motion of individual particle. The discrete element method (DEM) is one of the important examples of discrete model in simulating particulate flows. Simple flows are well established using the discrete element method. The interaction of 
all the particles with their environment and with each other is modeled in this method which is followed by calculating their trajectories, orientations and spins [6]. A broad spectrum of industrial problems have been discussed using two dimensional (2D) and three dimensional (3D) DEM simulations [7].

The discrete approach discussed above is further mainly categorized into two approaches for the simulation of particulate flow, (i) the Eulerian approach (ii) and the Lagrangian approach. In Eulerian approach, the whole domain is covered by the fluid and uses a fixed background mesh which is independent of particle parameters. Based on this approach, Joseph and Glowinski proposed a technique [8]. In Lagrangian approach, the concept of a moving mesh is used in which the nodes move along with the motion of the particle boundary. A modification of this approach is referred to as Arbitrary Lagrangian Eulerian Approach (ALE) [9]. Eulerian approach has the advantage that the mesh remains constant and focuses to get high numerical approximation through acceptable computational cost. On the other hand, Lagrangian approach gives very acuurate results but the simulation cost is high. In the technique proposed by Glowinski and Singh [10], FEM based background grid supports the particle contained fluid domain in Eulerian approach where the solid particles are treated separately through Newton-Euler equations. The complete solution of Navier-Stokes equations for the coupled system of fluid and particle is achieved using the fictitious boundary method (FBM) [11], [12], [13], [14], [15] such that the the fluid and particles interfaces are viewed as additional constraints for an internal boundary in the fluid domain retaining the same computational domain which remains fixed. On the other hand, computational grid is reshaped in ALE approach and the mesh nodes are accumulated more near the fluid particle interface and the process is moved forward with adopting a replaced mesh. A particular handling of such a method is discussed using the distributed Lagrange multiplier method (DLM) and the fictitious boundary method [16], [17], [18] developed by Glowinski [8]. A stress field in the particle domain, introduced by Patankar et al. [19] combined with the DLM method, removed the necessity to calculate the translational and angular velocity of the moving particles. Sangani introduced a DLM based formulation using a control volume method [20] for steady stokes flow. Abbasi et al. [21], [22] analyzed the phenomenon when multiple structures are in wake of one another, the mechanism depends upon the adjustment, gap distance, shape and size of structures. Inoue et al.[23] implanted a technique based on finite difference method in order to find the solution of 2D unsteady compressible Navier-Stokes equation and analyzed the wake achieved by two square obstacles arranged on staggered positions by considering space effect between them in a uniform flow at low Mach numbers. Wang et al.[24] considered computational mesh as a horizontal soap film tunnel along with two circular obstacles placed in staggered positions.

In order to prevent the complicated collisions of the particles with each other, channel wall and internal obstacles, a true definition for the collision model [27] is needed because collision or near collision of the solid rigid particles can cause extreme problems in case the rigid particles are very close to each other and thus remarkably increase the evaluation and cost of simulation [28]. Recently, Usman [29] presented a brief comparison of distinct collision models for circular rigid particles in a two dimensional framework in fluid channel. In other observations, researchers have concluded that for rough particles, physical touching might happen which may consequently effect the dependent motion of the rigid solid particles [30]. Ardekani and Rangel [31] used distributed Lagrange multiplier approach to simulate unsteady motion and collision of two particles in fluid having a dilute suspension with control volume approach [32]. A simple algorithm to simulate colliding particles has been discussed by Patankar [33].

The present study focuses on the numerical investigations for the rapid change in drag forces on the interior obstacles while the particle crosses the obstacles and change in the motion of fluid and its behavior due to interacting falling circular particle. Impact of wall of the domain and obstacles on the trajectories of the particle are also studied in fluid flow. Fictitious boundary method [34] is acquired to achieve the solution of viscous incompressible fluid flow and the moving solid particles, which is a direct simulation technique. The well known Eulerian technique, which is independent of particle shape, number of particles and particle size, is used to solve the problem of fluid and particle system in particulate flow.

\section{Mathematical Modeling}

Consider fluid flow along with a solid particle having mass $M_{s}$ and density $\rho_{s} . \rho_{f}$ is the fluid density and $v$ represents the fluid viscosity. The domain occupied by the fluid is $\Omega_{f}$ and the domain occupied by the particle is $\Omega_{s}$ and $\partial \Omega_{s}$ represents boundary of the particle. Hence, the total domain is given by $\Omega_{T}=\Omega_{f} \cup \Omega_{s}$

\subsection{Incompressible Fluid and Particle Motion}

The motion of incompressible fluid in the domain $\Omega_{f}$ is governed by the Navier-Stokes equations [35], [36] 
$\rho_{f}\left(\frac{\partial \boldsymbol{u}}{\partial t}+\boldsymbol{u} \cdot \nabla \boldsymbol{u}\right)-\nabla \cdot \sigma=0, \quad \nabla \cdot \boldsymbol{u}=0 \quad \forall t \in(0, T)$,

where $\sigma$ is the total stress tensor in the fluid phase, defined as,

$\sigma=-p \boldsymbol{I}+\mu_{f}\left[\nabla \boldsymbol{u}+(\nabla \boldsymbol{u})^{T}\right]$.

Here, fluid velocity is $\boldsymbol{u}, p$ is the pressure, coefficient of viscosity is $\mu_{f}$ and $\boldsymbol{I}$ is the identity tensor.

The translational and rotational motion of the freely moving rigid particle in fluid is due to the hydrodynamic forces, collision forces due to particle and outer wall and particle-cylinder interaction and gravitational acceleration. The Newton-Euler equations, in this case, takes the form

$M_{s} \frac{d \boldsymbol{U}_{s}}{d t}=\left(\triangle M_{s}\right) \boldsymbol{g}+\boldsymbol{F}_{s}+\boldsymbol{F}_{s}^{\prime}, \quad \boldsymbol{I}_{s} \frac{d \omega_{s}}{d t}+\omega_{s} \times\left(\boldsymbol{I}_{s} \omega_{s}\right)=\boldsymbol{T}_{s}$.

$\boldsymbol{U}_{s}$ and $\omega_{s}$ respectively are the translational and angular velocities of the particle, $M_{s}$ is the particle mass and we write $\triangle M_{s}=M_{s}-M_{f}$, where $M_{f}$ is the mass occupied by the fluid in the same volume as $M_{s}$. Drag and lift forces acting on the particle are represented by $\boldsymbol{F}_{s}, \boldsymbol{F}_{s}^{\prime}$ are the particle collision forces, the moment of inertia tensor and the resultant torque acting about the center of mass of the particle is $\boldsymbol{I}_{s}$ and $\boldsymbol{T}_{s}$ respectively and $\boldsymbol{g}$ denotes the gravitational acceleration.

The position $\boldsymbol{X}_{s}$ of the center of mass of the particle and its angle $\theta_{s}$ can be obtained after integrating the following kinematic equations [37], [38],

$\frac{d \boldsymbol{X}_{s}}{d t}=\boldsymbol{U}_{s}, \quad \frac{d \theta_{s}}{d t}=\omega_{s}$.

\subsection{Drag and Lift Hydrodynamic Forces and Torque}

The drag and lift forces $\boldsymbol{F}_{s}$ and the torque $\boldsymbol{T}_{s}$ acting on the mass center of the particle can be obtained by [39]

$\boldsymbol{F}_{s}=(-1) \int_{\partial \Omega_{s}}(\sigma \cdot \boldsymbol{n}) d \Gamma_{s}, \quad \boldsymbol{T}_{s}=(-1) \int_{\partial \Omega_{s}}\left(\boldsymbol{X}-\boldsymbol{X}_{s}\right) \times(\sigma \cdot \boldsymbol{n}) d \Gamma_{s}$.

where the unit vector $n$ acts normal to the boundary $\partial \Omega_{s}$ of the particle. Once the drag force is calculated, the drag and lift coefficients can be found using

$C_{d}=\frac{2 F_{D}}{\rho U^{2} D}, \quad C_{l}=\frac{2 F_{L}}{\rho U^{2} D}$

where $U$ and $D$ is the characteristic velocity and length respectively.

\subsection{Fluid-Particle Coupling using Fictitious Boundary Method}

At the fluid and particle interface $\partial \Omega_{s}$, no-slip boundary conditions are applied and the velocity $\boldsymbol{u}_{s}(\boldsymbol{X}) \forall \boldsymbol{X} \in \overline{\boldsymbol{\Omega}}_{s}$ is given by,

$\boldsymbol{u}_{s}(\boldsymbol{X})=\boldsymbol{U}_{s}+\omega_{s} \times\left(\boldsymbol{X}-\boldsymbol{X}_{s}\right)$.

The fictitious boundary method (FBM) works over a multigrid finite element method by incorporating the particle domain within the fluid domain. The additional constraints arising due to the particle's boundary motion at the particle-fluid interface are included in the Navier-Stokes equations, by extending the fluid domain with the combined fluid and particle domain, which takes the form,

$$
\left\{\begin{array}{lr}
\nabla . u=0 & \forall X \in \Omega_{T} \\
\rho_{f}\left(\frac{\partial \boldsymbol{u}}{\partial t}+\boldsymbol{u} . \nabla \boldsymbol{u}\right)-\nabla . \sigma=0 & \forall X \in \Omega_{f} \\
\boldsymbol{u}(\boldsymbol{X})=\boldsymbol{U}_{s}+\omega_{s} \times\left(\boldsymbol{X}-\boldsymbol{X}_{s}\right) & \forall X \in \bar{\Omega}_{s} .
\end{array}\right.
$$




\subsection{Particle Collisions}

We will use a collision model for the calculation of particle-wall collision forces $F_{s}^{W}$ presented by Glowinski, Joseph, Singh and coauthors [10] and present a modified model for the particle-cylinder collision forces $F_{s}^{c}$. Therefore, $\boldsymbol{F}_{s}^{\prime}=\boldsymbol{F}_{s}^{c}+\boldsymbol{F}_{s}^{W}$.

\section{Particle-Cylinder Collision Model}

We have introduced a small change in the collision model for interacting particle and cylinder proposed in [10], that is, we have considered the circular cylinder as a second particle and hence the model takes the form

$\boldsymbol{F}_{s}^{c}=\left\{\begin{array}{lll}0, & \text { for } \quad D_{s, c}>R_{s}+R_{c}+\rho, \\ \frac{1}{\varepsilon_{p}}\left(\boldsymbol{X}_{s}-\boldsymbol{X}_{c}\right)\left(R_{s}+R_{c}+\rho-D_{s, c}\right)^{2}, & \text { for } \quad R_{s}+R_{c} \leq D_{s, c} \leq R_{s}+R_{c}+\rho \\ \frac{1}{\varepsilon_{p}^{\prime}}\left(\boldsymbol{X}_{s}-\boldsymbol{X}_{c}\right)\left(R_{s}+R_{c}-D_{s, c}\right), & \text { for } \quad D_{s, c} \leq R_{s}+R_{c}\end{array}\right.$

the coordinates of the center of the cylinder are $X_{c}$ and $R_{S}$ and $R_{c}$ denotes the radius of particle and cylinder respectively. The distance between $\boldsymbol{X}_{s}$ and $\boldsymbol{X}_{c}$ is $D_{s, c}=\left|\boldsymbol{X}_{s}-\boldsymbol{X}_{c}\right| . \rho$ is the minimum distance to activate the force of repulsion between particle and cylinder and is taken one mesh element apart. Values for the positive stiffness parameters $\varepsilon_{p}$ and $\varepsilon_{p}^{\prime}$ are chosen as such to avoid discontinuity or singularity.

\section{Particle-Wall Collision Model}

For particle-wall collision model, the corresponding model is expressed by [10]

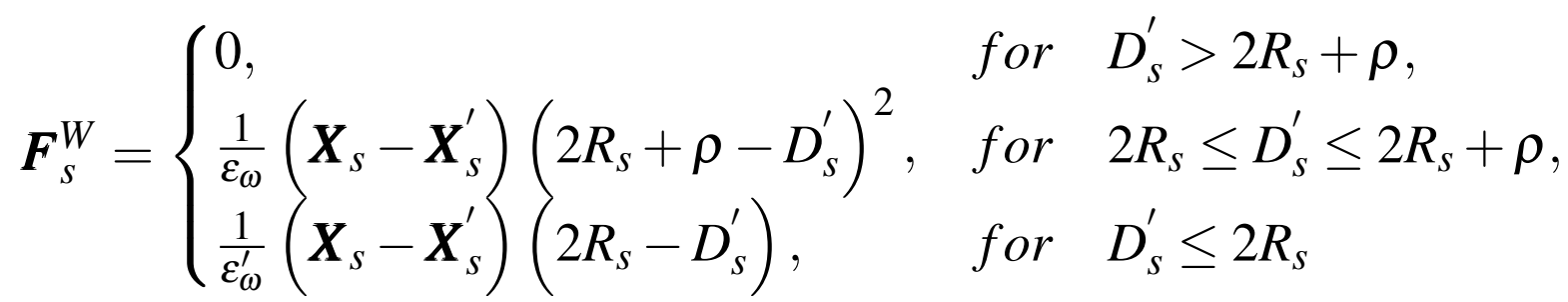

where $\boldsymbol{X}_{s}^{\prime}$ is the coordinate of the center of mass of the nearest imaginary particle $p_{s}^{\prime}$ imagined on the boundary wall with respect to the particle. $D_{s}^{\prime}=\left|\boldsymbol{X}_{s}-\boldsymbol{X}_{s}^{\prime}\right|$ is the distance between the center of the imaginary particle $p_{s}^{\prime}$ and the mass center of particle. $\varepsilon_{w}$ and $\varepsilon_{w}^{\prime}$ are small positive stiffness parameters for particle-wall collisions, usually their values can be taken as $\varepsilon_{w}=\frac{\varepsilon_{p}}{2}$ and $\varepsilon_{w}^{\prime}=\frac{\varepsilon_{p}^{\prime}}{2}$ in the calculations.

\section{Numerical Results}

We have examined the behavior of a falling particle inside a vertical channel passing across four internal circular obstacles. When the falling particle crosses the obstacles, it disturbs the pressure field and consequently disturbs the fluid motion as well as the hydrodynamic drag and lift forces acting on the surface of obstacles. The disturbance propagates further when particle move forward. The collisions and overlapping of particle with obstacles and with the outer wall are avoided using collision models discussed in equation (2.9) and equation (2.10). The width and height of the computational channel is 3 and 15 as shown in figure 3.1a and 3.1b. The moving rigid 2D particle has density $\rho_{s}=1.25$. Density of incompressible fluid is taken $\rho_{f}=1.0$ and the Reynolds number is $R e=100$. In numerical simulations, we consider the particle of radius $R=0.25$. We consider that initially at $t=0$ the fluid and particle are both at rest. The falling motion is started only due to the gravitational acceleration $g=980$. Zero dirichlet boundary conditions at the walls of the channel are assumed. The simulations are performed on fixed equidistant meshes using CFD code FEATFLOW [40].

The simulations are performed on mesh refinement level-5 comprising of 146, 432 number of elements. Mesh independence is guaranteed at refinement level-5. The experiments are performed keeping three different initial positions of the particle in $x$-direction while retaining the same $y$ position. First three cases shown in figure 3.1 are performed keeping the cylinder configuration given by Coarse mesh 1 where the upper three cylinders are fixed (see figure 3.1), the cases 4-6 are performed keeping the cylinder configuration given by Coarse mesh 2 again keeping the upper three cylinders fixed. A variety of 
configurations for the position of fourth obstacle are selected comprising of inline obstacles arrangement and staggered obstacles arrangement with each other. Moreover, the study includes effect of increasing space between two obstacles by moving the fourth obstacle along the $y$-direction at three different positions and expanding the gap between them.

Different images in figure 3.1 show the position of particle at different time and the disturbance it creates while falling down and crossing the obstacles. Obstacles are placed in an inline arrangement as shown in figure 3.1c, figure 3.1d and figure 3.1e, similarly Obstacles are placed in an staggered arrangement as shown in figure $3.1 \mathrm{f}$, figure $3.1 \mathrm{~g}$ and figure $3.1 \mathrm{~h}$ while the initial $\mathrm{x}$-positions of particle are $1.50,1.55$ and 1.60 respectively for all the cases. The particle is shown colliding and crossing the first and second cylinder in figure $3.1 \mathrm{~d}$ and figure $3.1 \mathrm{e}$ respectively.

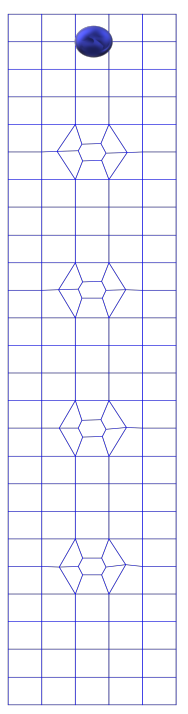

(a) Coarse mesh 1

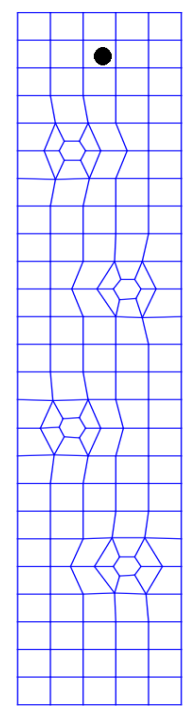

(b) Coarse mesh 2

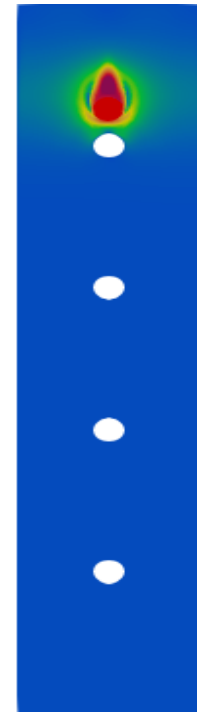

(c) Case i

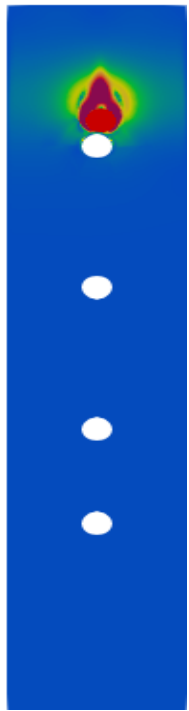

(d) Case ii

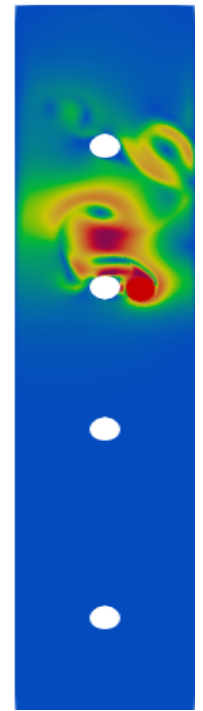

(e) Case iii

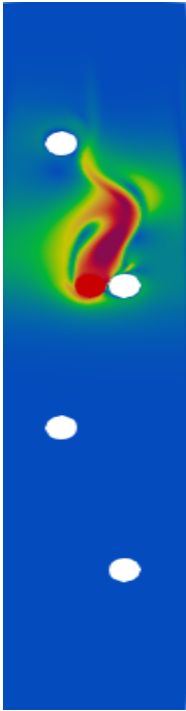

(f) Case iv

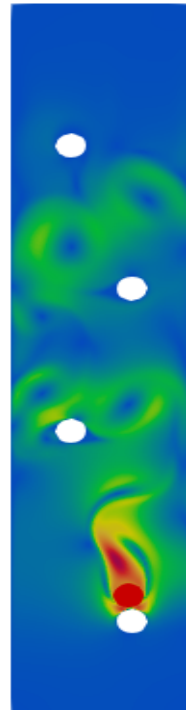

(g) Case v

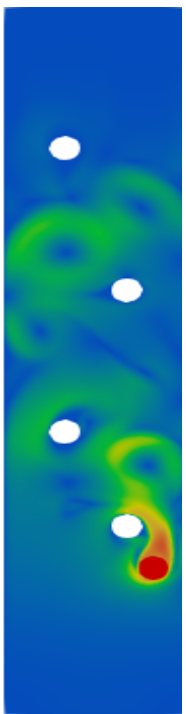

(h) Case vi

Figure 3.1: Schematic of different meshes with obstacles

Different images in figure 3.1 show the position of particle at different time and the disturbance it creates while falling down and passing the obstacles. These are total 6 cases in which we have found numerical results and simulations are performed, circular obstacles placed in inline and staggered arrangement are as follows:

(i) $(1.50,12.0),(1.50,9.0),(1.50,6.0),(1.50,3.0) \quad$ (ii) $(1.50,12.0),(1.50,9.0),(1.50,6.0),(1.50,4.0)$ (iii) $(1.50,12.0),(1.50,9.0),(1.50,6.0),(1.50,2.0) \quad$ (iv) $(1.0,12.0),(2.0,9.0),(1.0,6.0),(2.0,3.0)$ (v) $(1.0,12.0),(2.0,9.0),(1.0,6.0),(2.0,2.0) \quad(\mathrm{vi})(1.0,12.0),(2.0,9.0),(1.0,6.0),(2.0,4.0)$
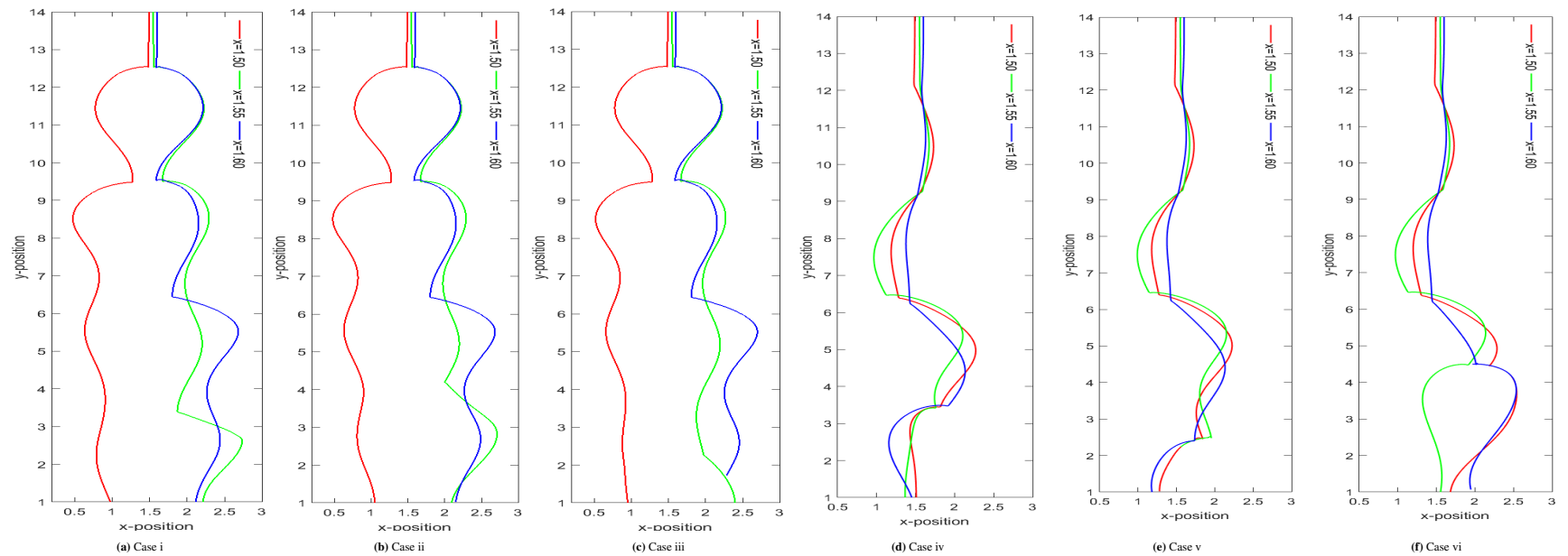

Figure 3.2: Trajectories of particle passing and crossing cylinders

Figure 3.2 shows trajectories of the falling particle starting from different initial positions and passing the cylinders through the course. $C_{1}$ and $C_{2}$ denote the first and second cylinder respectively. Similarly, $C_{1 x}$ and $C_{1 y}$ respectively represents the x-position and y-position of the first cylinder. 
Six meshes have been adopted having different arrangements of the cylinders for the study. In first three meshes, $C_{1 x}$ is fixed at position (1.5) and in meshes 4-6, $C_{1 x}$ is fixed at position (1.0). $C_{4 y}$ is taken at three positions 2.0, 3.0 and 4.0 for all the cases. Figure 3.2(a) shows results when the cylinders are inline i.e. $C_{1}$ at position $(1.5,12.0)$ and $C_{4}$ at position $(1.5,3.0)$ and the simulations are performed by taking three particle starting positions $(1.50,14.0),(1.55,14.0)$ and $(1.60,14.0)$ shown in green, red and blue lines respectively. It has been observed that when the starting position of the particle is $(1.50,14.0)$, it always passes from the left side of the first cylinder and may pass from left or right of the fourth cylinder depending on the gap between the cylinders. $C_{1 x}=1.50$ ensures that for the other two starting particle positions except the starting particle position $(1.5,14.0)$, the particle passes from the right side of the first cylinder and for $C_{1 x}=1.50$ the particle always passes from the left side of the first cylinder as noticeable from figure 3.2. It has been concluded that the gap between the cylinders, starting position of the particle and the inline/staggered arrangement of cylinders plays an important role while defining the paths for the particles and the fluid motion.

Table 1: Case 1 with 4 obstacles

\begin{tabular}{c|ccc|c|c|c}
\hline \hline Particle & \multicolumn{2}{|c|}{$C_{d}$ on $C_{2}$} & \multicolumn{2}{c|}{$C_{d}$ on $C_{3}$} & \multirow{2}{*}{$t_{2}$} & \multirow{2}{*}{$t_{4}$} \\
\cline { 2 - 5 } Position & $\min$ & $\max$ & $\min$ & $\max$ & & \\
\hline \hline 1.50 & -3.3472 & 2.7136 & -3.3150 & 2.1469 & 0.192 & 0.093 \\
1.55 & -1.8778 & 4.3367 & -3.9430 & 4.1884 & 0.165 & 0.198 \\
1.60 & -0.5700 & 5.0199 & -3.6266 & 2.2387 & 0.177 & 0.105 \\
\hline
\end{tabular}

\begin{tabular}{c|ccc|c|c|c|c}
\hline \hline Particle & \multicolumn{2}{|c|}{$S_{2}$} & \multicolumn{2}{c|}{$S_{3}$} & \multicolumn{2}{c|}{$S_{4}$} \\
\cline { 2 - 7 } Position & $\min$ & $\max$ & $\min$ & $\max$ & $\min$ & $\max$ \\
\hline \hline 1.50 & 0.4779 & $\overline{-}$ & 0.6363 & $\overline{-}$ & 0.8006 & $\overline{-}$ \\
1.55 & - & 2.2925 & - & 2.1755 & - & 2.7369 \\
1.60 & - & 2.5116 & - & 2.6818 & - & 2.4408 \\
\hline
\end{tabular}

Table 2: Case 2 with 4 obstacles

\begin{tabular}{c|c|c|c|c|c|c}
\hline \hline \multirow{2}{*}{$\begin{array}{c}\text { Particle } \\
\text { Position }\end{array}$} & \multicolumn{2}{|c|}{$C_{d}$ on $C_{2}$} & \multicolumn{2}{c|}{$C_{d}$ on $C_{3}$} & \multirow{2}{*}{$t_{2}$} & \multirow{2}{*}{$t_{4}$} \\
\cline { 2 - 5 } & $\min$ & $\max$ & $\min$ & $\max$ & & \\
\hline \hline 1.50 & -3.3138 & 2.7005 & -3.6266 & 2.2387 & 0.192 & 0.093 \\
1.55 & -1.8694 & 4.3658 & -3.8777 & 4.1629 & 0.168 & 0.120 \\
1.60 & -0.5084 & 5.0170 & -3.6239 & 2.2037 & 0.105 & 0.177 \\
\hline
\end{tabular}

\begin{tabular}{c|ccc|c|c|c|c}
\hline \hline Particle & \multicolumn{2}{|c|}{$S_{2}$} & \multicolumn{2}{c|}{$S_{3}$} & \multicolumn{2}{c|}{$S_{4}$} \\
\cline { 2 - 7 } Position & $\min$ & $\max$ & $\min$ & $\max$ & $\min$ & $\max$ \\
\hline \hline 1.50 & 0.4819 & $-\overline{-}$ & 0.6339 & $\overline{-}$ & 0.8313 & $\overline{-}$ \\
1.55 & - & 2.2899 & - & 2.1800 & - & 2.3979 \\
1.60 & - & 2.1510 & - & 2.6828 & - & 2.3500 \\
\hline
\end{tabular}

Table 3: Case 3 with 4 obstacles

\begin{tabular}{c|cc|c|c|c|c}
\hline \hline Particle & \multicolumn{2}{|c|}{$C_{d}$ on $C_{2}$} & \multicolumn{2}{c|}{$C_{d}$ on $C_{3}$} & \multirow{2}{*}{$t_{2}$} & \multirow{2}{*}{$t_{4}$} \\
\cline { 2 - 5 } Position & $\min$ & $\max$ & $\min$ & $\max$ & & \\
\hline \hline 1.50 & -3.3834 & 2.6160 & -3.4913 & 2.2619 & 0.186 & 0.093 \\
1.55 & -1.8245 & 4.5054 & -4.0316 & 4.3156 & 0.165 & 0.108 \\
1.60 & -0.6283 & 5.0387 & -3.7709 & 2.3287 & 0.108 & 0.093 \\
\hline
\end{tabular}

\begin{tabular}{c|ccc|c|c|c|c}
\hline \hline Particle & \multicolumn{2}{|c|}{$S_{2}$} & \multicolumn{2}{c|}{$S_{3}$} & \multicolumn{2}{c|}{$S_{4}$} \\
\cline { 2 - 7 } Position & $\min$ & $\max$ & $\min$ & $\max$ & $\min$ & $\max$ \\
\hline \hline 1.50 & 0.5213 & $\overline{-}$ & 0.6595 & $\overline{-}$ & 0.8372 & $-\overline{-}$ \\
1.55 & - & 2.2714 & - & 2.1733 & - & 2.0932 \\
1.60 & - & 2.1500 & - & 2.6977 & - & 2.3490 \\
\hline
\end{tabular}

Table 4: Case 4 with 4 Obstacles

\begin{tabular}{c|cc|c|c|c|c}
\hline \hline Particle & \multicolumn{2}{|c|}{$C_{d}$ on $C_{2}$} & \multicolumn{2}{c|}{$C_{d}$ on $C_{3}$} & \multirow{2}{*}{$t_{2}$} & \multirow{2}{*}{$t_{4}$} \\
\cline { 2 - 5 } Position & $\min$ & $\max$ & $\min$ & $\max$ & & \\
\hline \hline 1.50 & -4.9426 & 5.4015 & -4.4172 & 4.8132 & 0.096 & 0.165 \\
1.55 & -4.8121 & 6.1664 & -1.9383 & 2.8117 & 0.105 & 0.171 \\
1.60 & -4.4132 & 5.6758 & -6.0258 & 3.9727 & 0.093 & 0.144 \\
\hline
\end{tabular}




\begin{tabular}{c|c|c|c|c|ccc}
\hline \hline Particle & \multicolumn{2}{|c|}{$S_{2}$} & \multicolumn{2}{c|}{$S_{3}$} & \multicolumn{2}{c}{$S_{4}$} \\
\cline { 2 - 7 } Position & $\min$ & $\max$ & $\min$ & $\max$ & & $\max$ \\
\hline \hline 1.50 & 1.2951 & - & - & 2.1394 & 1.4285 & - \\
1.55 & 1.1770 & - & - & 2.0960 & 1.4406 & - \\
1.60 & 1.4125 & - & - & 1.8115 & 1.1587 & - \\
\hline
\end{tabular}

Table 5: Case 5 with 4 Obstacles

\begin{tabular}{c|c|c|c|c|c|c}
\hline \hline Particle & \multicolumn{2}{|c|}{$C_{d}$ on $C_{2}$} & \multicolumn{2}{c|}{$C_{d}$ on $C_{3}$} & \multirow{2}{*}{$t_{2}$} & \multirow{2}{*}{$t_{4}$} \\
\cline { 2 - 6 } Position & $\min$ & $\max$ & $\min$ & $\max$ & & \\
\hline \hline 1.50 & -4.9213 & 5.4056 & -4.3006 & 4.9239 & 0.099 & 0.189 \\
1.55 & -4.1258 & 6.2198 & -2.2576 & 2.9974 & 0.105 & 0.216 \\
1.60 & -4.4145 & 5.6588 & -5.9977 & 4.3494 & 0.093 & 0.153 \\
\hline
\end{tabular}

\begin{tabular}{c|c|c|c|c|c|c}
\hline \hline Particle & \multicolumn{2}{|c|}{$S_{2}$} & \multicolumn{2}{c|}{$S_{3}$} & \multicolumn{2}{c}{$S_{4}$} \\
\cline { 2 - 7 } Position & $\min$ & $\max$ & $\min$ & $\max$ & & $\max$ \\
\hline \hline 1.50 & 1.2910 & - & - & 2.1067 & 1.3332 & - \\
1.55 & 1.1987 & - & - & 2.1362 & 1.2790 & - \\
1.60 & 1.4129 & - & - & 1.8115 & 1.2014 & - \\
\hline
\end{tabular}

Table 6: Case 6 with 4 Obstacles

\begin{tabular}{c|cc|c|c|c|c}
\hline \hline Particle & \multicolumn{2}{|c|}{$C_{d}$ on $C_{2}$} & \multicolumn{2}{c|}{$C_{d}$ on $C_{3}$} & \multirow{2}{*}{$t_{2}$} & \multirow{2}{*}{$t_{4}$} \\
\cline { 2 - 5 } Position & $\min$ & $\max$ & $\min$ & $\max$ & & \\
\hline \hline 1.50 & -4.9618 & 5.3961 & -4.7092 & 4.8608 & 0.096 & 0.198 \\
1.55 & -4.4861 & 6.1392 & -2.0728 & 2.7670 & 0.105 & 0.144 \\
1.60 & -4.4402 & 5.6673 & -6.0445 & 2.7578 & 0.093 & 0.159 \\
\hline
\end{tabular}

\begin{tabular}{c|c|c|c|c|c|c}
\hline \hline Particle & \multicolumn{2}{|c|}{$S_{2}$} & \multicolumn{3}{c|}{$S_{3}$} & \multicolumn{2}{c}{$S_{4}$} \\
\cline { 2 - 7 } Position & $\min$ & $\max$ & $\min$ & $\max$ & & $\max$ \\
\hline \hline 1.50 & 1.3020 & - & - & 2.1230 & 2.5394 & - \\
1.55 & 1.1777 & - & - & 2.1258 & 1.3216 & - \\
1.60 & 1.4186 & - & - & 1.7423 & - & 2.5337 \\
\hline
\end{tabular}

In table 1 , table 2 , table 3 , table 4 , table 5 and table $6, t_{1}$ and $t_{2}$ denote the time taken by the falling particle when it comes in contact or interacts and crosses $C_{1}$ and $C_{2}$ respectively. $s_{1}$ and $s_{2}$ denote the minimum or maximum x-shift achieved by the particle while crossing $C_{1}$ and $C_{2}$ respectively. More time is taken by the particle to pass the cylinder if it collides while passing the cylinder and takes less time if it passes without touching the cylinder. The fluid behavior across the circular cylinders also affects the motion of the particle and contributes whether the particle will collide or not with the cylinder. If the x-shift attained by the particle is more than 0.83 or less than 0.47 while passing and crossing the cylinder then it has collided with the cylinder otherwise it suggests that the particle has passed without colliding the cylinder.

First two columns in figure 3.3 shows fluctuations in the drag coefficient when particle is passing by $C_{1}$ and $C_{2}$. It can be seen that when the particle starts interacting with $C_{1}$ and $C_{2}$, the drag values instantly shoots up to a very high value and after crossing the cylinder, gradually reaches its mean value (see figure 3.3(a) and figure 3.3(b)). The third column of graphs in figure 3.3 shows oscillations of drag coefficient for cylinder 3 . It has been observed that the drag on $C_{2}$ fluctuates when the particles passes across $C_{1}$ and disturbs the fluid motion. Figure 3.3 shows some selected cases for different configurations of cylinders. Figure 3.3(a-c) presents result for inline cylinders case when $C_{1}$ and $C_{4}$ are at positions $(1.5,12.0)$ and $(1.5,3.0)$ with particle starting position 1.50. Figure 3.3(d-f) presents result for inline cylinders case when $C_{1}$ and $C_{4}$ are at positions $(1.5,12.0)$ and $(1.5,4.0)$ with particle starting position 1.55. Figure 3.3(g-i) presents result for staggered cylinders case when $C_{1}$ and $C_{4}$ are at positions $(1.0,12.0)$ and $(2.0,3.0)$ with particle starting position 1.60. Figure 3.3(j-1) presents result for staggered cylinders case when $C_{1}$ and $C_{4}$ are at position $(1.0,12.0)$ and $(2.0,2.0)$ with particle starting position at 1.55 . Figure $3.3(\mathrm{~m}-\mathrm{o})$ presents result for inline cylinders case when $C_{1}$ and $C_{4}$ are at positions $(1.50,12.0)$ and $(1.5,2.0)$ with particle starting position 1.60. Other cases exhibit a similar pattern and are hence not shown. 
8

International Journal of Emerging Multidiciplinaries
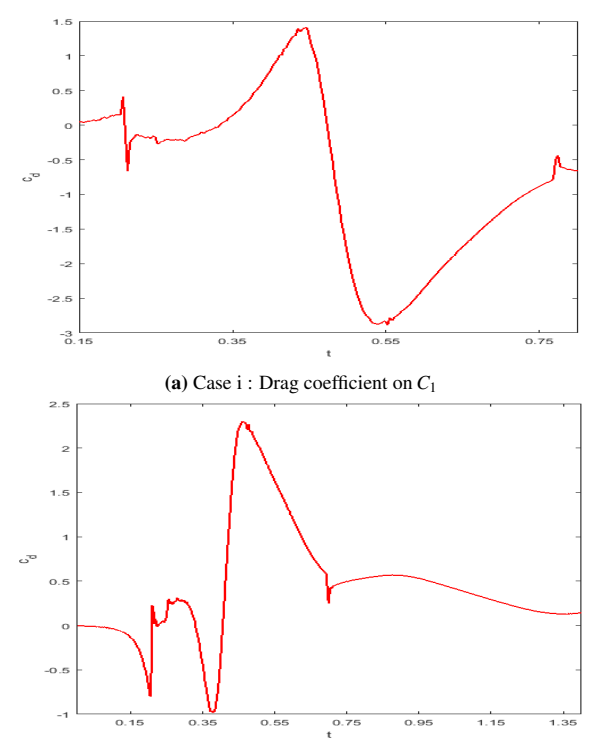

(d) Case ii : Drag coefficient on $C_{1}$

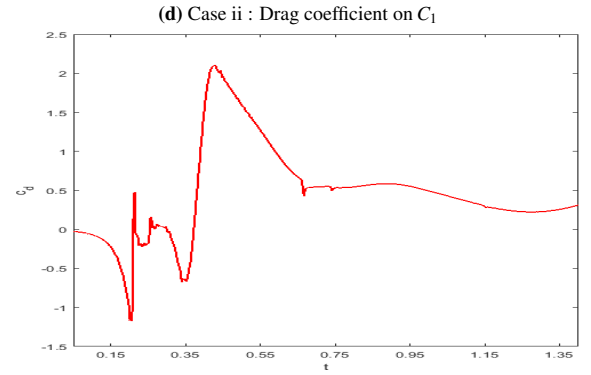

(g) Case iii : Drag coefficient on $C_{1}$

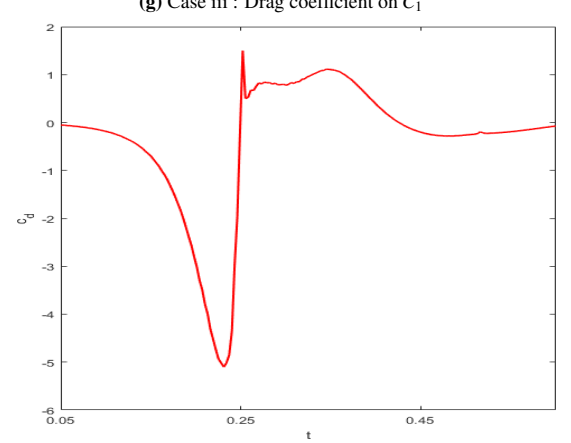

(j) Case iv : Drag coefficient on $C_{1}$

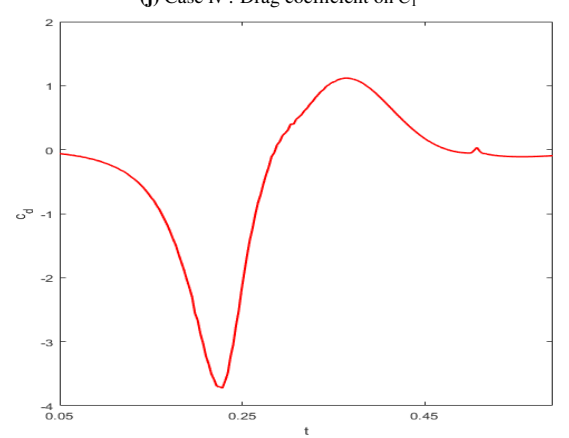

(m) Case v : Drag coefficient on $C_{1}$

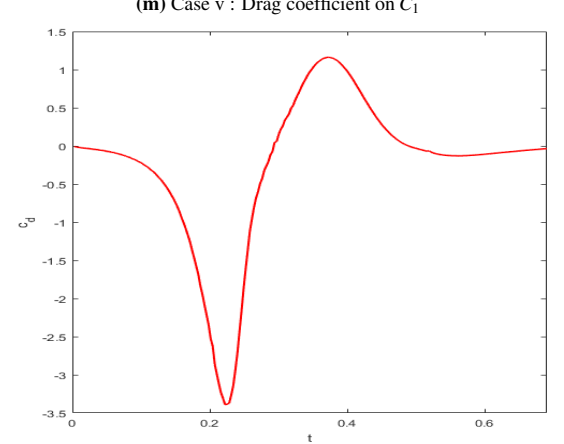

(p) Case vi : Drag coefficient on $C_{1}$

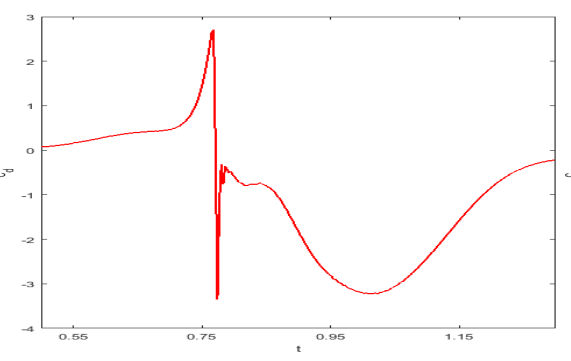

(b) Case i : Drag coefficient on $C_{2}$

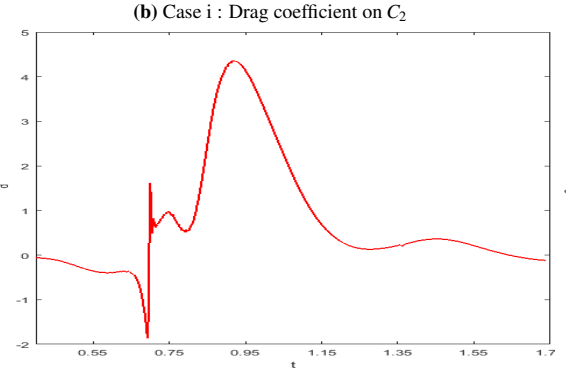

(e) Case ii : Drag coefficient on $C_{2}$

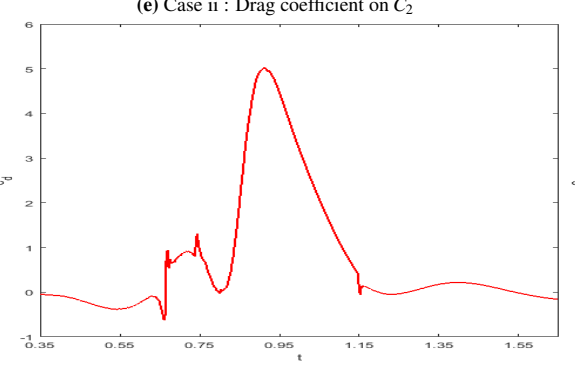

(h) Case iii : Drag coefficient on $C_{2}$

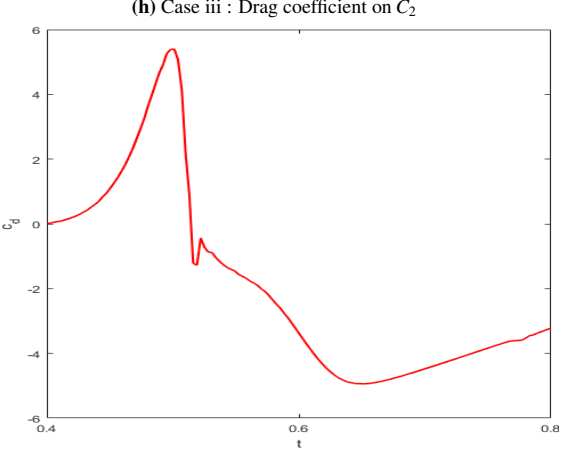

(k) Case iv : Drag coefficient on $C_{2}$

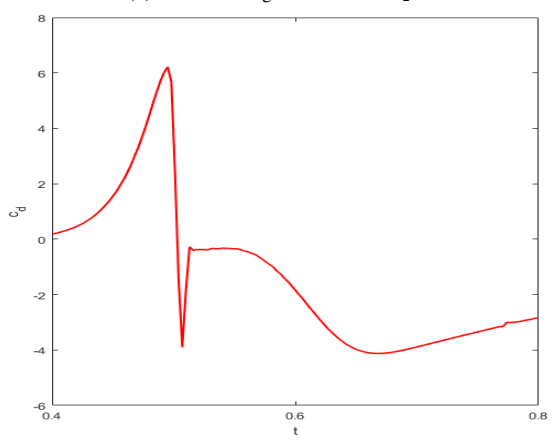

(n) Case v : Drag coefficient on $C_{2}$

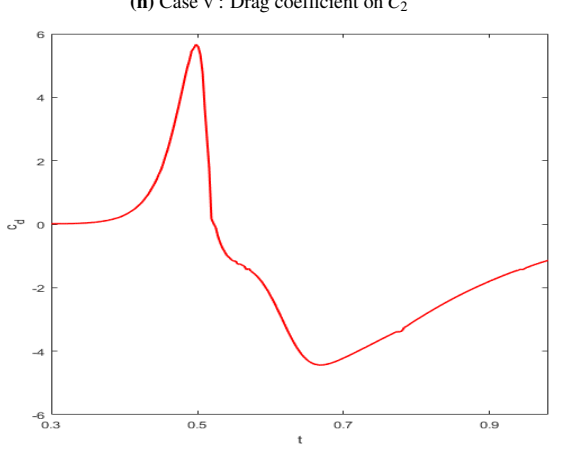

(q) Case vi : Drag coefficient on $C_{2}$

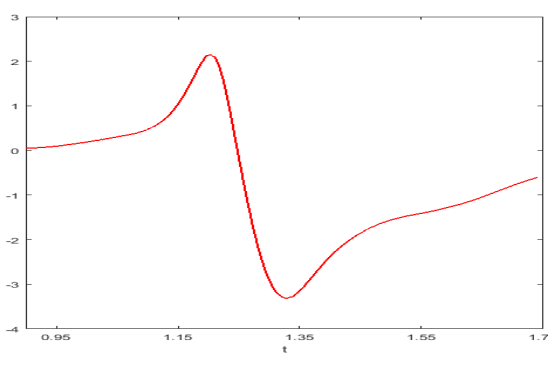

(c) Case i : Drag coefficient on $C_{3}$

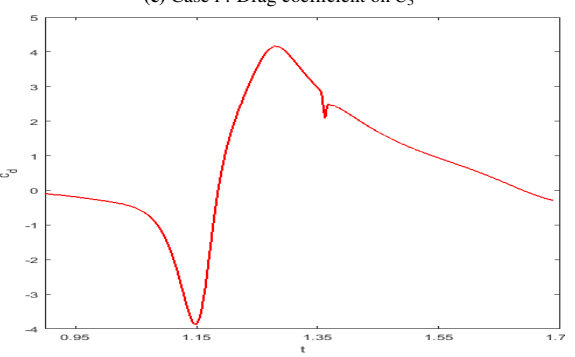

(f) Case ii : Drag coefficient on $C_{3}$

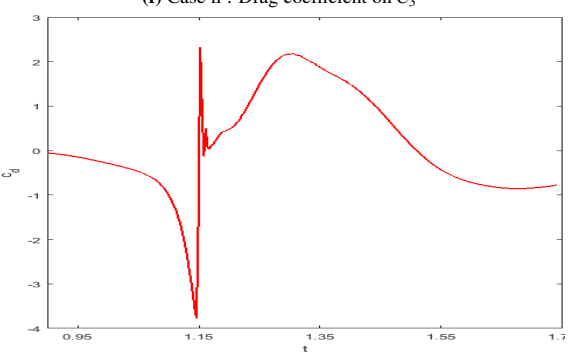

(i) Case iii : Drag coefficient on $C_{3}$

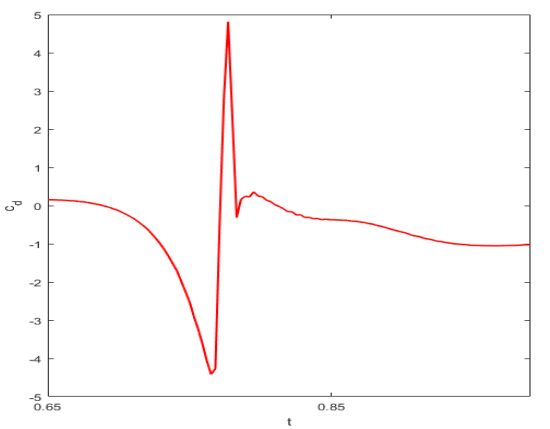

(l) Case iv : Drag coefficient on $C_{3}$
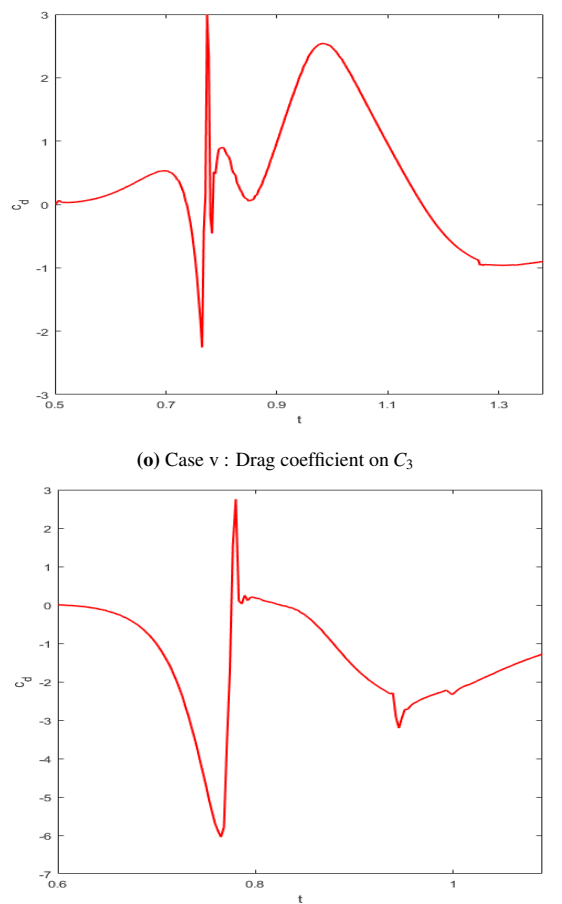

(r) Case vi : Drag coefficient on $C_{3}$

Figure 3.3: Drag on $C_{1}, C_{2}$ and $C_{3}$ with gap 0.5 
Drag1 and Drag2 displays the maximum and minimum values of drag coefficient in table 1, table 2, table 3 , table 4 , table 5 and table 6 when the particle is crosses $C_{1}$ and $C_{2}$ respectively. Drag $2 \_1$ denotes the drag on $C_{2}$ when particle is crossing $C_{1}$. The drag values on cylinders $C_{1}$ and $C_{2}$ changes rapidly as soon as the particle reaches near the cylinder and starts crossing it. The drag values are more extreme if the particle crosses the cylinder while colliding as shown in table 1 , table 2 , table 3 , table 4 , table 5 and table 6. The table shows that if the maximum drag value is above 7.5 or minimum drag value is below -7.0 then the particle has collided while crossing the cylinder $C_{1}$ or $C_{2}$. The drag 'Drag 2_1' on $C_{2}$ due to $C_{1}$ when particle is crossing $C_{1}$ shows fluctuations because the disturbance created in the fluid motion due to particle crossing $C_{1}$ spreads till and beyond $C_{2}$. The gap distance between cylinders and cylinder's arrangement plays an important role in determining the drag 'Drag 2_1' on $C_{2}$ when particle crosses $C_{1}$.

\section{Conclusion}

In this research work, we have performed numerical technique of a direct simulation method to stimulate the particulate flow by using FBM. We have examined the behavior of falling particle under the action of gravitational acceleration, the collision and interaction of particle with four obstacles within a fluid flow channel. We have also discussed the behavior of obstacles. The first obstacle is fixed and the fourth obstacle is placed at three different positions along y-direction. A solid particle which is falling from three different positions, is interacting and colliding with all four obstacles during the course. We have calculated the numerical results on the fixed computational mesh with refinement level- 5 by taking three different initial positions of particle. During the fall of rigid particle, we have examined the behavior of fluid inside a channel while passing across the obstacles. We have examined this behavior using different configuration of the circular obstacles, inline and staggered arrangements of obstacles which showed different fluid behavior in a channel.

A specific collision model is also used for the collision of the particle and circular obstacles. Benefit of this collision model is that it can treat the scenario when the particle interact and tend to slightly overlap the obstacles due to numerical errors.

\section{Nomenclature}

$\begin{array}{ll}M_{s} & \text { Mass of solid particle } \\ \rho_{s} & \text { Density of particle } \\ \rho_{f} & \text { Density of fluid } \\ v & \text { Fluid viscosity } \\ \mathbf{u} & \text { Velocity of fluid } \\ p & \text { Pressure } \\ \sigma & \text { Stress tensor } \\ \mathbf{I} & \text { Identity tensor } \\ \mathbf{U}_{\mathbf{s}} & \text { velocity of solid particle } \\ \mathbf{X}_{\mathbf{s}} & \text { Center of solid circular particle } \\ \theta_{s} & \text { Orientation of particle } \\ \omega_{s} & \text { Angular velocity of particle } \\ \mathbf{F}_{\mathbf{s}} & \text { Drag and lift forces acting on the particle } \\ \boldsymbol{F}_{s}^{\prime} & \text { Particle collision forces } \\ \mathbf{g} & \text { Gravitational acceleration } \\ C_{d} & \text { Drag coefficient } \\ \text { FBM } & \text { Fictitious boundary method } \\ \text { DNS } & \text { Direct numerical simulation } \\ \text { FEM } & \text { Finite element method } \\ \text { DLM } & \text { Distributed lagrange multiplier method } \\ \text { DEM } & \text { Discrete element method } \\ \text { CFD } & \text { Computational fluid dynamics } \\ \text { ALE } & \text { Arbitrary lagrangian eulerian approach } \\ & \end{array}$

\section{Acknowledgments}

The authors would like to thank the anonymous reviewers for their comments and suggestions. 


\section{References}

[1] A. R. Koblitz, S. Lovett, N. Nikiforakis, and W. D. Henshaw. Direct numerical simulation of particulate flows with an overset grid method. Journal of Computational Physics. 343, $414-431$ (2017).

[2] Santiago D. Costarelli, Luciano Garelli, Marcela A. Cruchaga, Mario A. Storti, Ronald Ausensi, and Sergio R. Idelsohn. An embedded strategy for the analysis of fluid structure interaction problems. Computer Methods in Applied Mechanics and Engineering. 300,106 - 128 (2016).

[3] Sydney Chapman and T. G. Cowling. The mathematical theory of non-uniform gases. American Journal of Physics. 30(5), 389-389 (1962).

[4] K. Kesava Rao and Prabhu R. Nott. An Introduction to Granular Flow. Cambridge Series in Chemical Engineering. Cambridge University Press, 2008.

[5] Andriy Sokolov, Ramzan Ali, and Stefan Turek. An afc-stabilized implicit finite element method for partial differential equations on evolving-in-time surfaces. Journal of Computational and Applied Mathematics. 289, $101-115$ (2015). Sixth International Conference on Advanced Computational Methods in Engineering (ACOMEN 2014).

[6] C S Campbell. Rapid granular flows. Annual Review of Fluid Mechanics, 22(1):57-90 (1990).

[7] Paul W. Cleary. Dem simulation of industrial particle flows: case studies of dragline excavators, mixing in tumblers and centrifugal mills. Powder Technology. 109(1), $83-104$ (2000).

[8] R. Glowinski, T. W. Pan, T. I. Hesla, and D. D. Joseph. A distributed lagrange multiplier/fictitious domain method for particulate flows. International Journal of Multiphase Flow. 25(5), 755 - 794 (1999).

[9] D. Wan and S. Turek. Fictitious boundary and moving mesh methods for the numerical simulation of rigid particulate flows. Journal of Computational Physics. 222(1), 28 - 56 (2007).

[10] N. A. Patankar, P. Singh, D. D. Joseph, R. Glowinski, and T. W. Pan. A new formulation of the distributed lagrange multiplier/fictitious domain method for particulate flows. Int. J. Multiphase Flow. 26, 1509-1524 (2000).

[11] Khuram Walayat, Zekun Wang, Kamran Usman, and Moubin Liu. An efficient multi-grid finite element fictitious boundary method for particulate flows with thermal convection. International Journal of Heat and Mass Transfer. 126, 452 - 465 (2018).

[12] K. Usman, K. Walayat, R. Mahmood, and N. Kousar. Analysis of solid particles falling down and interacting in a channel with sedimentation using fictitious boundary method. AIP Advances. 8(6), 065201 (2018).

[13] Khuram Walayat, Zhilang Zhang, Kamran Usman, Jianzhong Chang, and Moubin Liu. Dynamics of elliptic particle sedimentation with thermal convection. Physics of Fluids. 30(10), 103301 (2018).

[14] Khuram Walayat, Zhilang Zhang, Kamran Usman, Jianzhong Chang, and Moubin Liu. Fully resolved simulations of thermal convective suspensions of elliptic particles using a multigrid fictitious boundary method. International Journal of Heat and Mass Transfer. 139, 802 - 821 (2019).

[15] Saqia Jabeen, Kamran Usman, and Khuram Walayat. Numerical investigations for a chain of particles settling in a channel. Computational Particle Mechanics, Oct 2019.

[16] K Usman, K Walayat, R Mahmood, and N Kousar. Analysis of solid particles falling down and interacting in a channel with sedimentation using fictitious boundary method. AIP Advances. 8(6), 065201 (2018).

[17] Khuram Walayat, Zekun Wang, Kamran Usman, and Moubin Liu. An efficient multi-grid finite element fictitious boundary method for particulate flows with thermal convection. International Journal of Heat and Mass Transfer. 126, 452-465 (2018).

[18] Khuram Walayat, Zhilang Zhang, Kamran Usman, Jianzhong Chang, and Moubin Liu. Dynamics of elliptic particle sedimentation with thermal convection. Physics of Fluids. 30(10), 103301 (2018).

[19] Neelesh A Patankar, Pushpendra Singh, Daniel D Joseph, Roland Glowinski, and T-W Pan. A new formulation of the distributed lagrange multiplier/fictitious domain method for particulate flows. International Journal of Multiphase Flow. 26(9), 1509-1524 (2000).

[20] Ashok S Sangani and Guobiao Mo. Inclusion of lubrication forces in dynamic simulations. Physics of fluids. 6(5), 1653-1662 (1994).

[21] Waqas Sarwar Abbasi, Shams-ul Islam, Hamid Rahman, and Raheela Manzoor. Numerical investigation of fluid-solid interaction for flow around three square cylinders. AIP Advances. 8(2), 025221 (2018).

[22] Kaman Usman, Junaid Asmat, Waqas Sarwar Abbasi, Saqia Jabeen, and Jabbar Ali. Analysis of a floating particle interacting with fixed cylinders inside a channel. Journal of the Brazilian Society of Mechanical Sciences and Engineering, 2021.

[23] O Inoue, M Mori, and N Hatakeyama. Aeolian tones radiated from flow past two square cylinders in tandem. Physics of Fluids. 18(4), 046101 (2006). 
[24] Si-ying Wang, Fang-bao Tian, Lai-bing Jia, Xi-yun Lu, and Xie-zhen Yin. Secondary vortex street in the wake of two tandem circular cylinders at low reynolds number. Physical Review E. 81(3), 036305 (2010).

[25] Anthony J. C. Ladd. Numerical simulations of particulate suspensions via a discretized boltzmann equation. part 1. theoretical foundation. Journal of fluid mechanics. 271, 285-309 (1994).

[26] John F Brady and Georges Bossis. Stokesian dynamics. Annual review of fluid mechanics. 20(1), 111-157 (1988).

[27] Decheng Wan and Stefan Turek. An efficient multigrid-fem method for the simulation of solid-liquid two phase flows. Journal of Computational and Applied Mathematics. 203(2), 561 - 580 (2007).

[28] Lin Jian-Zhong, Wang Ye-Long, and James A Olsen. Sedimentation of rigid cylindrical particles with mechanical contacts. Chinese Physics Letters. 22(3), 628 (2005).

[29] Kamran Usman. Numerical Analysis of Collision Models in 2D Particulate Flow. PhD thesis, Technische Universitaet Dortmund, Fakultaet fuer Mathematik, 2013.

[30] P. A. Arp and S. G. Mason. The kinetics of flowing dispersions: Ix. doublets of rigid spheres (experimental). Journal of colloid and interface science. 61(1), 44-61 (1977).

[31] A. M. Ardekani and R. H. Rangel. Unsteady motion of two solid spheres in stokes flow. Physics of Fluids. 18(10), 103306 (2006).

[32] A. M. Ardekani and R. H. Rangel. Numerical investigation of particle-particle and particle-wall collisions in a viscous fluid. Journal of Fluid Mechanics. 596, 437-466 (2008).

[33] Suhas Patankar. Numerical heat transfer and fluid flow. CRC press, 2018.

[34] Stefan Turek, Decheng Wan, and Liudmila S Rivkind. The fictitious boundary method for the implicit treatment of dirichlet boundary conditions with applications to incompressible flow simulations. In Challenges in Scientific Computing-CISC 2002, Springer. 37-68 (2003).

[35] V. John. Higher order finite element methods and multigrid solvers in a benchmark problem for the 3d navier-stokes equations. Int. J. for Numerical Methods in Fluids. 40, 775-798 (2002).

[36] Stefan Turek. Numerical Analysis of a New Time-stepping $\theta$-scheme for Incompressible Flow Simulations. Ergebnisberichte angewandte Mathematik. Univ. Dortmund, Fachbereich Mathematik, 2005.

[37] Decheng Wan and Stefan Turek. Direct numerical simulation of particulate flow via multigrid fem techniques and the fictitious boundary method. International Journal for Numerical Methods in Fluids. 51(5), 531-566 (2006).

[38] Decheng Wan, Stefan Turek, and Liudmila S. Rivkind. An efficient multigrid fem solution technique for incompressible flow with moving rigid bodies. In Miloslav Feistauer, Vit Dolejsi, Petr Knobloch, and Karel Najzar, editors, Numerical Mathematics and Advanced Applications, Springer Berlin Heidelberg. 844-853 (2004).

[39] S. Kim and S.J. Karrila. Microhydrodynamics: Principles and Selected Applications. Butterworth Heinemann series in chemical engineering. Dover Publications, 2005.

[40] S. Turek. Featflow. finite element software for the incompressible navier-stokes equations: User manual, release 1.1. Technical report, 1998. 